Supplement of Atmos. Chem. Phys. Discuss., 15, 22715-22779, 2015

http://www.atmos-chem-phys-discuss.net/15/22715/2015/

doi:10.5194/acpd-15-22715-2015-supplement

(C) Author(s) 2015. CC Attribution 3.0 License.

(c) (i)

\title{
Investigation of error sources in regional inverse estimates of greenhouse gas emissions in Canada
}

\section{E. Chan et al.}

Correspondence to: E. Chan (elton.chan@ec.gc.ca)

The copyright of individual parts of the supplement might differ from the CC-BY 3.0 licence. 


\section{Supplementary information}

Table S1. Results of the synthetic data experiments presented in Table 3. Table S1, S3 and Table S2, S4 correspond to results using the MCMC and CFM estimation methods respectively. The monthly and annual (Y2009) estimation biases (relative percentage differences from the target) are presented, i.e. (posterior estimate - target)/target $\mathrm{x}$ 100\%. The standard deviations (YSTD) of the monthly estimation biases are shown in the last column. Color codes in red, orange, yellow, gray, light blue, and dark blue represent relative percentage differences that are greater than 100, between 50 and 100, between 20 and 50, between -10 and -25, between 25 and -50 , and less than -50 respectively. 
Table S1 (MCMC).

Province Experiment Jan Feb Mar Apr May Jun Jul Aug Sep Oct Nov Dec Y2009 YSTD $\mathrm{AB} / \mathrm{SK} \quad 1$

$\mathrm{AB} / \mathrm{SK} \quad 2$

\begin{tabular}{llllll|l|l|l|l|l|l|l|l|}
0 & 0 & 0 & -0 & 0 & 0 & 0 & -0 & 0 & 0 & 0 & 0 & 0 & 0
\end{tabular}

$\mathrm{AB} / \mathrm{SK} \quad 3$

$\begin{array}{llllllllllllll}0 & 0 & 0 & -0 & 0 & -0 & -0 & 0 & -0 & 0 & 0 & 0 & 0 & 0\end{array}$

$\mathrm{AB} / \mathrm{SK} \quad 4$

$\mathrm{AB} / \mathrm{SK} \quad 5$

$\begin{array}{llllllllllllll}0 & 0 & 0 & -0 & 0 & 0 & -0 & 0 & 0 & 0 & 0 & 0 & 0 & 0\end{array}$

$\mathrm{AB} / \mathrm{SK} \quad 6$

AB/SK 7

$\mathrm{AB} / \mathrm{SK} \quad 8$

$\begin{array}{llll}0 & 0 & 0 & 0\end{array}$

$\mathrm{AB} / \mathrm{SK} \quad 9$

$\begin{array}{llllllllllllll}0 & 0 & 0 & -0 & 0 & 0 & -0 & -0 & -0 & -0 & 0 & 0 & 0 & 0\end{array}$

$\mathrm{AB} / \mathrm{SK} \quad 10$

$\begin{array}{llll}0 & 0 & 0 & -0\end{array}$

$\begin{array}{lll}-0 & 0 & 0\end{array}$

$\begin{array}{lll}-0 & 0 & 0\end{array}$

$-0$

$\begin{array}{lll}0 & 0 & -0\end{array}$

$\mathrm{AB} / \mathrm{SK} \quad 11$

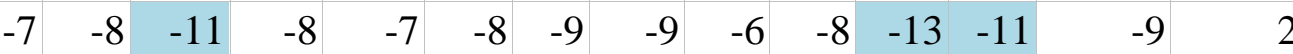

$\mathrm{AB} / \mathrm{SK} \quad 12$

$\begin{array}{llll}-4 & -6 & -7 & -7\end{array}$

$\mathrm{AB} / \mathrm{SK} \quad 13$

$\begin{array}{lll}-4 & -5 & -8\end{array}$

$\mathrm{AB} / \mathrm{SK} \quad 14$

$\begin{array}{llll}-4 & -3 & -5 & -10 \\ -3 & -4 & -6 & -2\end{array}$

AB/SK 15

$-3 \quad-4 \quad-6$

AB/SK 16

$\begin{array}{lll}-4 & -5 & -6\end{array}$

$\mathrm{AB} / \mathrm{SK} \quad 17$

$\begin{array}{llllllllllllll}-5 & -5 & -7 & -11 & -10 & -11 & -9 & -8 & -10 & -8 & -9 & -5\end{array}$

AB/SK 18

$\begin{array}{llll}-1 & 60 & 65 & 18\end{array}$

AB/SK 19

$-1 \quad 55$

$58 \quad 75$

46

$8-46$

$-2 \quad 54$

$57 \quad 88$

$\begin{array}{lll}41 \quad 16 & -32\end{array}$

12

26

$$
51
$$

57

16

$\begin{array}{lllll}7 & 58 & 46 & 67 & 19\end{array}$

$27 \quad 32$

AB/SK 20

$-4 \quad 58$

$\begin{array}{lll}47 & -9 & -16\end{array}$

$\begin{array}{lllll}6 & 82 & 42 & 60 & 13\end{array}$

$34 \quad 32$

AB/SK 21

$10 \quad 51 \quad 36$

AB/SK 22

$148 \quad 46$

AB/SK 23

$\begin{array}{lll}-5 & 47 \quad 34\end{array}$

$73 \quad 51$
67

$\begin{array}{lll}51 & 15 & -19\end{array}$

$-3 \quad 93$

$\begin{array}{lll}41 & 53 & 16\end{array}$

$35 \quad 36$

AB/SK 24

$46 \quad 50$

87

$5-10$

$\begin{array}{llll}60 & 38 & 54 & 18\end{array}$

$36 \quad 34$

$30 \quad 30$

(1)

$-5 \quad 47$

AB/SK 25 $\begin{array}{lll}-6 & 48 & 39\end{array}$

AB/SK 26

$\begin{array}{llllllll}-2 & 44 & 36 & 77 & 14 & 6 & \mathbf{- 5 3}\end{array}$

$-2 \quad 54$

439

$37 \quad 11$

26

25 25

AB/SK 27

$-9 \quad 29$

AB/SK 28

$-14 \quad 37$

AB/SK 29

$\begin{array}{lll}-9 & 39 & 38\end{array}$

AB/SK 30

$-1137$

$\mathrm{AB} / \mathrm{SK} \quad 31$

$\begin{array}{lll}-15 & 31 \quad 21\end{array}$

$\begin{array}{lllll}44 & 38 & 0 & -3 & -23\end{array}$

288

$0 \quad 72$

$88 \quad 30$

20

$\begin{array}{llll}29 & 4 & 25 & 35\end{array}$

$\begin{array}{llll}26 & 2 & 20 & 35\end{array}$

$\begin{array}{llllll}32 & 24 & -31 & -27 & \mathbf{- 5 3} & -7\end{array}$

$\begin{array}{lll}2 & 8 & 36\end{array}$

27

$13 \quad 22$

\begin{tabular}{lllll|l|l}
-7 & 32 & 27 & 26 & -1 & 5 & 30
\end{tabular}

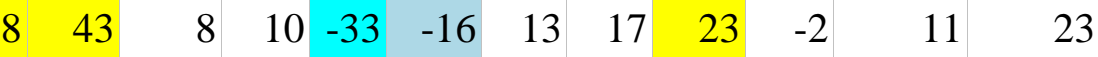

\begin{tabular}{|l|l|l|l|l|l|l|l|l|l|l|l|}
23 & 31 & -17 & 4 & -28 & -25 & 45 & 21 & 12 & -3 & 8 & 24 \\
\hline
\end{tabular}

\begin{tabular}{llllllllll|l|l|}
21 & 33 & 5 & -25 & -44 & -10 & 25 & 16 & 10 & -7 & 4 & 24
\end{tabular} 
Table S2 (CFM).

Province Experiment Jan Feb Mar Apr May Jun Jul Aug Sep Oct Nov Dec Y2009 YSTD \begin{tabular}{|l|l|l|l|l|l|l|l|l|l|l|l|l|l|l|l|}
\hline $\mathrm{AB} / \mathrm{SK}$ & 32 & 1 & 1 & 3 & 21 & 36 & 30 & 20 & 18 & 21 & 8 & 4 & 1 & 13 & 12 \\
\hline
\end{tabular}

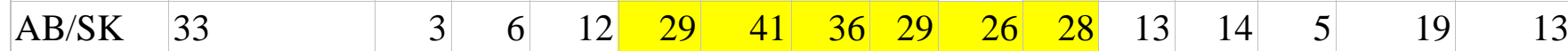

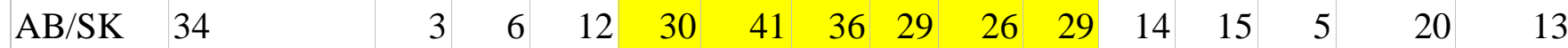

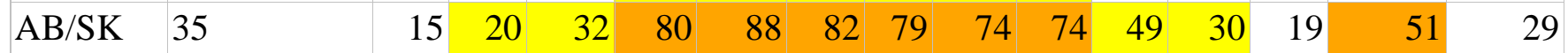

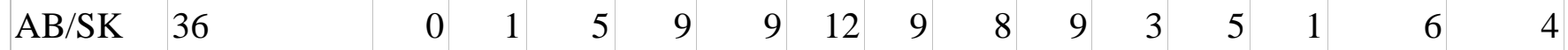
\begin{tabular}{|l|l|l|l|l|l|l|l|l|l|l|l|l|l|l|l|}
$\mathrm{AB} / \mathrm{SK}$ & 37 & 1 & 2 & 7 & 12 & 13 & 16 & 12 & 11 & 14 & 5 & 8 & 2 & 8 & 5 \\
\hline
\end{tabular} \begin{tabular}{|l|l|l|l|l|l|l|l|l|l|l|l|l|l|l|l|}
\hline $\mathrm{AB} / \mathrm{SK}$ & 38 & 1 & 3 & 9 & 18 & 19 & 23 & 17 & 17 & 17 & 8 & 9 & 2 & 11 & 7 \\
\hline
\end{tabular} \begin{tabular}{|l|l|l|l|l|l|l|l|l|l|l|l|l|l|l|l|}
\hline $\mathrm{AB} / \mathrm{SK}$ & 39 & 3 & 3 & 11 & 22 & 27 & 32 & 23 & 17 & 25 & 12 & 13 & 3 & 15 & 10 \\
\hline
\end{tabular} \begin{tabular}{|l|l|l|l|l|l|l|l|l|l|l|l|l|l|l|l|}
$\mathrm{AB} / \mathrm{SK}$ & 40 & 6 & 6 & 14 & 26 & 29 & 35 & 30 & 21 & 30 & 16 & 17 & 5 & 19 & 10
\end{tabular}

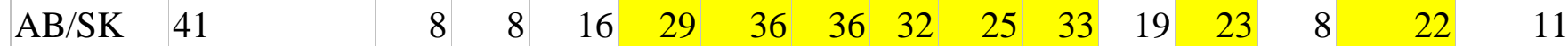

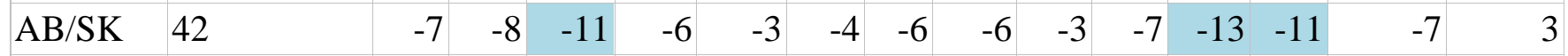
\begin{tabular}{|l|l|l|l|l|l|l|l|l|l|l|l|l|l|l|l|}
$\mathrm{AB} / \mathrm{SK}$ & 43 & -4 & -5 & -7 & -2 & -2 & -1 & -3 & -3 & -1 & -5 & -7 & -7 & -4 & 2
\end{tabular} \begin{tabular}{|l|l|l|l|l|l|l|l|l|l|l|l|l|l|l|l|}
$\mathrm{AB} / \mathrm{SK}$ & 44 & -4 & -5 & -7 & -1 & 1 & 3 & -0 & -4 & 2 & -4 & -8 & -6 & -3 & 4
\end{tabular} \begin{tabular}{|l|l|l|l|l|l|l|l|l|l|l|l|l|l|l|l|}
$\mathrm{AB} / \mathrm{SK}$ & 45 & -3 & -2 & -2 & 2 & 6 & 7 & 4 & 2 & 5 & -2 & -6 & -5 & 0 & 4 \\
\hline
\end{tabular}

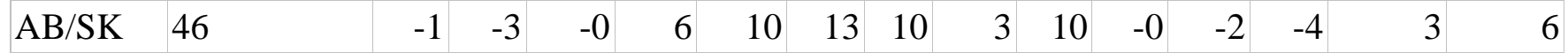
\begin{tabular}{|l|l|l|l|l|l|l|l|l|l|l|l|l|l|l|l|}
\hline $\mathrm{AB} / \mathrm{SK}$ & 47 & 1 & -1 & 1 & 8 & 13 & 15 & 12 & 6 & 12 & 2 & -0 & -3 & 5 & 6 \\
\hline
\end{tabular} \begin{tabular}{|l|l|l|l|l|l|l|l|l|l|l|l|l|l|l|l|}
\hline $\mathrm{AB} / \mathrm{SK}$ & 48 & 0 & -0 & 4 & 10 & 16 & 14 & 15 & 10 & 14 & 3 & 3 & -0 & 7 & 6
\end{tabular}

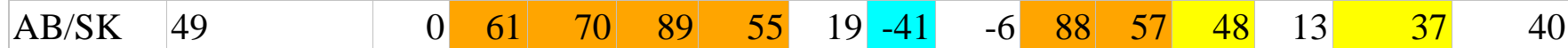

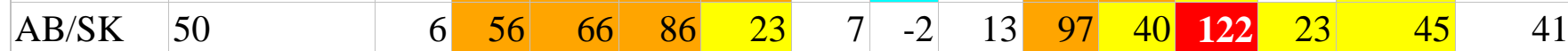

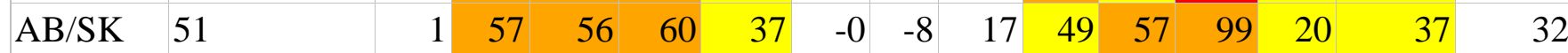

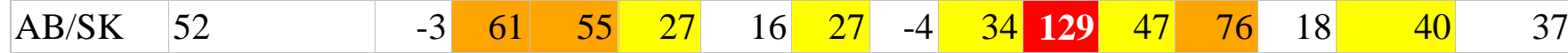

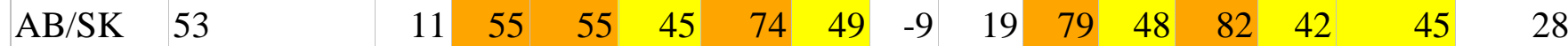

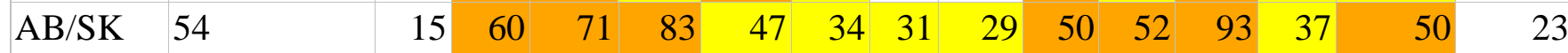

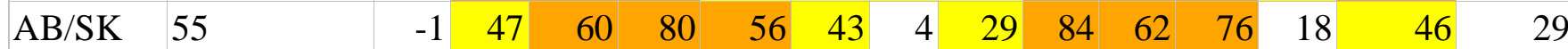

\begin{tabular}{|c|c|c|c|c|c|c|c|c|c|c|c|c|c|c|c|}
\hline $\mathrm{AB} / \mathrm{SK}$ & 56 & -6 & 52 & 53 & 52 & 1 & 12 & -22 & 10 & 103 & 26 & 38 & 8 & 27 & 34 \\
\hline $\mathrm{AB} / \mathrm{SK}$ & 57 & -2 & 51 & 41 & 57 & 24 & -2 & -32 & 21 & 62 & 31 & 51 & 7 & 26 & 29 \\
\hline $\mathrm{AB} / \mathrm{SK}$ & 58 & -5 & 42 & 44 & 50 & 34 & -12 & 0 & 1 & 41 & 40 & 47 & 15 & 25 & 23 \\
\hline $\mathrm{AB} / \mathrm{SK}$ & 59 & -11 & 50 & 55 & 71 & 30 & 16 & -28 & 3 & 59 & 40 & 51 & 8 & 29 & 31 \\
\hline $\mathrm{AB} / \mathrm{SK}$ & 60 & 1 & 55 & 54 & 44 & 25 & 6 & -7 & -0 & 62 & 32 & 56 & 26 & 30 & \\
\hline $\mathrm{AB} / \mathrm{SK}$ & 61 & -0 & 44 & 56 & 62 & 44 & 19 & -23 & 8 & 66 & 41 & 52 & 25 & 33 & 27 \\
\hline $\mathrm{AB} / \mathrm{SK}$ & 62 & -4 & 39 & 28 & 44 & 2 & 10 & -7 & 12 & 57 & 44 & 39 & 11 & 23 & \\
\hline
\end{tabular}


Table S3 (MCMC).

Province Experiment Jan Feb Mar Apr May Jun Jul Aug Sep Oct Nov Dec Y2009 YSTD \begin{tabular}{ll|lllllllllllllr}
$\mathrm{ON}$ & 1 & 0 & 0 & 0 & 0 & 0 & 0 & 0 & 0 & 0 & 0 & 0 & 0 & 0 & 0
\end{tabular}

\begin{tabular}{ll|llllllllllllll}
$\mathrm{ON}$ & 2 & 0 & -0 & 0 & 0 & 0 & 0 & 0 & 0 & 0 & 0 & 0 & 0 & 0 & 0
\end{tabular}

\begin{tabular}{ll|llllllllllllll}
$\mathrm{ON}$ & 3 & 0 & 0 & 0 & 0 & -0 & 0 & 0 & 0 & 0 & 0 & 0 & 0 & 0 & 0
\end{tabular}

\begin{tabular}{ll|llllllllllllll}
$\mathrm{ON}$ & 4 & 0 & -0 & 0 & 0 & 0 & 0 & 0 & -0 & 0 & 0 & 0 & 0 & 0 & 0
\end{tabular}

\begin{tabular}{ll|llllllllllllll}
$\mathrm{ON}$ & 5 & 0 & 0 & 0 & 0 & -0 & 0 & 0 & -0 & 0 & 0 & -0 & 0 & 0 & 0
\end{tabular}

\begin{tabular}{ll|llllllllllllll}
$\mathrm{ON}$ & 6 & 0 & 0 & 0 & 0 & -0 & 0 & -0 & -0 & -0 & 0 & 0 & 0 & 0 & 0
\end{tabular}

\begin{tabular}{ll|rrrrrrrrrrrrrr}
\hline $\mathrm{ON}$ & 7 & 0 & 0 & 0 & 0 & 0 & 0 & -0 & -0 & 0 & 0 & 0 & 0 & 0 & 0
\end{tabular}

\begin{tabular}{ll|llllllllllllll}
$\mathrm{ON}$ & 8 & -0 & -0 & 0 & -0 & 0 & -0 & 0 & -0 & 0 & 0 & -0 & -0 & -0 & 0
\end{tabular}

\begin{tabular}{ll|llllllllllllll}
$\mathrm{ON}$ & 9 & -0 & 0 & -0 & -0 & -0 & 0 & -0 & 0 & 0 & 0 & 1 & -0 & 0 & 0
\end{tabular}

\begin{tabular}{ll|llllllllllllll}
$\mathrm{ON}$ & 10 & 0 & 1 & 0 & -0 & 0 & 1 & 0 & 0 & 0 & 0 & 0 & 0 & 0 & 0
\end{tabular}

\begin{tabular}{|c|c|c|c|c|c|c|c|c|c|c|c|c|c|c|c|}
\hline ON & 11 & -16 & -19 & -16 & -18 & -18 & -18 & -17 & -14 & -17 & -18 & -18 & -15 & -17 & 1 \\
\hline ON & 12 & -8 & -7 & -5 & -11 & -11 & -7 & -11 & -3 & -15 & -14 & -7 & -11 & -9 & 4 \\
\hline ON & 13 & -2 & -8 & 4 & -11 & -8 & 1 & -7 & 6 & -4 & -5 & -1 & -5 & -3 & 5 \\
\hline ON & 14 & -2 & -7 & 3 & -10 & -7 & -1 & -7 & 4 & -4 & -5 & -1 & -5 & -4 & 4 \\
\hline ON & 15 & -2 & -2 & 2 & -5 & -5 & 1 & 1 & -1 & -4 & -2 & 1 & -7 & -2 & 3 \\
\hline ON & 16 & -2 & -3 & -1 & -4 & -4 & 0 & -1 & -2 & -4 & -3 & 0 & -6 & -3 & 2 \\
\hline ON & 17 & -2 & -3 & -3 & -4 & -4 & -2 & -2 & -3 & -4 & -5 & -3 & -7 & -3 & 1 \\
\hline
\end{tabular}

\begin{tabular}{|c|c|c|c|c|c|c|c|c|c|c|c|c|c|c|c|}
\hline ON & 18 & 135 & 91 & 177 & 146 & -9 & -21 & 8 & 120 & 199 & 37 & 105 & 157 & 98 & 75 \\
\hline ON & 19 & 109 & 75 & 137 & 119 & -7 & -11 & 7 & 86 & 159 & 45 & 87 & 120 & 79 & 57 \\
\hline ON & 20 & 91 & 94 & 149 & 94 & 76 & 49 & 15 & 146 & 93 & 56 & 115 & 133 & 94 & 40 \\
\hline ON & 21 & 68 & 69 & 125 & 100 & 55 & 24 & -19 & 130 & 47 & 46 & 129 & 141 & 78 & 50 \\
\hline ON & 22 & 10 & 23 & 75 & 64 & 50 & 4 & -17 & 79 & 49 & 34 & 66 & 46 & 40 & 30 \\
\hline ON & 23 & 27 & 28 & 65 & 46 & 21 & -13 & -13 & 65 & 53 & 21 & 62 & 43 & 34 & 27 \\
\hline ON & 24 & 8 & 12 & 55 & 23 & 2 & -13 & -15 & 50 & 34 & 14 & 41 & 38 & 21 & 23 \\
\hline
\end{tabular}

\begin{tabular}{|c|c|c|c|c|c|c|c|c|c|c|c|c|c|c|c|}
\hline ON & 25 & 97 & 57 & 137 & 95 & -26 & -52 & -9 & 81 & 151 & 14 & 79 & 121 & 64 & 66 \\
\hline ON & 26 & 89 & 55 & 116 & 77 & -17 & -15 & -4 & 71 & 133 & 23 & 67 & 94 & 59 & 50 \\
\hline ON & 27 & 94 & 78 & 132 & 78 & 60 & 56 & -10 & 150 & 77 & 37 & 127 & 130 & 85 & 46 \\
\hline ON & 28 & 57 & 55 & 118 & 57 & 68 & 35 & -26 & 129 & 32 & 40 & 148 & 125 & 71 & 51 \\
\hline ON & 29 & -3 & 10 & 68 & 41 & 39 & 5 & -25 & 69 & 50 & 29 & 56 & 32 & 31 & 29 \\
\hline ON & 30 & 16 & 16 & 56 & 36 & 17 & -16 & -17 & 60 & 54 & 15 & 56 & 36 & 28 & 27 \\
\hline ON & 31 & -8 & 4 & 52 & 10 & 4 & -13 & -14 & 48 & 40 & 6 & 25 & 31 & 16 & 23 \\
\hline
\end{tabular}


Table S4 (CFM).

Province Experiment Jan Feb Mar Apr May Jun Jul Aug Sep Oct Nov Dec Y2009 YSTD

\begin{tabular}{|l|l|r|r|r|r|r|r|r|r|r|r|r|r|r|r|r|}
\hline ON & 32 & 0 & 0 & 1 & 1 & 1 & 1 & 1 & 1 & 1 & -0 & -0 & 1 & 1 & 1 \\
\hline ON & 33 & 15 & 21 & 22 & 20 & 23 & 17 & 19 & 22 & 20 & 9 & 14 & 16 & 18 & 4 \\
\hline ON & 34 & 15 & 20 & 21 & 21 & 24 & 18 & 20 & 23 & 19 & 9 & 13 & 16 & 18 & 4 \\
\hline ON & 35 & 27 & 27 & 29 & 34 & 30 & 26 & 29 & 30 & 28 & 23 & 26 & 28 & 28 & 3 \\
\hline ON & 36 & 3 & 7 & 9 & 8 & 16 & 6 & 7 & 12 & 6 & 2 & 2 & 4 & 7 & 4 \\
\hline ON & 37 & 6 & 10 & 14 & 22 & 20 & 14 & 14 & 16 & 16 & 7 & 4 & 11 & 13 & 5 \\
\hline ON & 38 & 8 & 12 & 16 & 24 & 21 & 15 & 16 & 18 & 17 & 9 & 5 & 13 & 14 & 5 \\
\hline ON & 39 & 17 & 28 & 25 & 38 & 29 & 30 & 28 & 27 & 38 & 16 & 14 & 29 & 26 & 8 \\
\hline ON & 40 & 26 & 37 & 32 & 48 & 35 & 39 & 36 & 32 & 47 & 21 & 20 & 37 & 34 & 9 \\
\hline ON & 41 & 38 & 46 & 38 & 58 & 43 & 47 & 43 & 39 & 54 & 30 & 30 & 43 & 42 & 8
\end{tabular}

\begin{tabular}{|l|l|r|r|r|r|r|r|r|r|r|r|r|r|r|r|r|r|r|}
\hline ON & 42 & -16 & -19 & -16 & -18 & -18 & -18 & -17 & -14 & -17 & -18 & -18 & -15 & -17 & 1 \\
\hline ON & 43 & -5 & -1 & 6 & -3 & 5 & -2 & -3 & 7 & -8 & -12 & -5 & -6 & -3 & 6 \\
\hline ON & 44 & 3 & 2 & 15 & 8 & 11 & 13 & 5 & 18 & 11 & -0 & 2 & 5 & 8 & 6 \\
\hline ON & 45 & 5 & 2 & 16 & 11 & 15 & 12 & 7 & 18 & 13 & 2 & 4 & 7 & 9 & 6 \\
\hline ON & 46 & 14 & 24 & 25 & 31 & 22 & 27 & 28 & 25 & 33 & 10 & 13 & 21 & 23 & 7 \\
\hline ON & 47 & 22 & 32 & 30 & 43 & 29 & 38 & 33 & 31 & 43 & 17 & 18 & 29 & 30 & 9 \\
\hline ON & 48 & 35 & 39 & 35 & 54 & 37 & 46 & 41 & 36 & 50 & 22 & 25 & 34 & 38 & 9 \\
\hline
\end{tabular}

\begin{tabular}{|l|l|r|r|r|r|r|r|r|r|r|r|r|r|r|r|}
\hline ON & 49 & $\mathbf{1 3 8}$ & 84 & $\mathbf{1 9 2}$ & $\mathbf{1 2 7}$ & -10 & -42 & 16 & $\mathbf{1 2 7}$ & $\mathbf{2 0 3}$ & 44 & $\mathbf{1 3 3}$ & $\mathbf{1 6 0}$ & $\mathbf{1 0 0}$ & $\mathbf{7 9}$ \\
\hline ON & 50 & $\mathbf{1 7 0}$ & $\mathbf{2 6 3}$ & $\mathbf{2 4 7}$ & $\mathbf{2 0 5}$ & -21 & 18 & -17 & 61 & $\mathbf{3 7 8}$ & $\mathbf{1 4 8}$ & $\mathbf{1 5 9}$ & $\mathbf{1 2 2}$ & $\mathbf{1 4 7}$ & 121 \\
\hline ON & 51 & $\mathbf{1 9 8}$ & $\mathbf{3 7 6}$ & $\mathbf{2 9 8}$ & $\mathbf{2 5 4}$ & $\mathbf{1 3 5}$ & $\mathbf{1 3 5}$ & -3 & $\mathbf{2 0 1}$ & $\mathbf{2 9 1}$ & $\mathbf{1 7 3}$ & $\mathbf{3 3 1}$ & $\mathbf{3 4 2}$ & $\mathbf{2 3 2}$ & 109 \\
\hline ON & 52 & $\mathbf{1 8 4}$ & $\mathbf{2 3 5}$ & $\mathbf{2 7 7}$ & $\mathbf{2 3 4}$ & $\mathbf{1 0 5}$ & $\mathbf{1 0 7}$ & -18 & $\mathbf{1 7 0}$ & $\mathbf{1 4 1}$ & $\mathbf{1 9 8}$ & $\mathbf{3 0 8}$ & $\mathbf{2 6 8}$ & $\mathbf{1 8 7}$ & 91 \\
\hline ON & 53 & 26 & 61 & $\mathbf{2 0 0}$ & $\mathbf{2 2 9}$ & $\mathbf{1 3 4}$ & 49 & -13 & $\mathbf{1 0 8}$ & $\mathbf{8 6}$ & $\mathbf{3 6 8}$ & $\mathbf{8 5}$ & 50 & $\mathbf{1 1 3}$ & 105 \\
\hline ON & 54 & 8 & 44 & $\mathbf{1 9 1}$ & $\mathbf{1 6 5}$ & $\mathbf{1 0 8}$ & 68 & 4 & $\mathbf{1 0 6}$ & $\mathbf{7 6}$ & $\mathbf{3 1 9}$ & $\mathbf{7 7}$ & 72 & $\mathbf{1 0 1}$ & 87 \\
\hline ON & 55 & -4 & 70 & $\mathbf{1 8 1}$ & $\mathbf{1 1 6}$ & $\mathbf{1 3 5}$ & 36 & 17 & 71 & $\mathbf{1 1 7}$ & $\mathbf{1 0 1}$ & $\mathbf{4 2}$ & 79 & 79 & 53 \\
\hline
\end{tabular}

\begin{tabular}{|c|c|c|c|c|c|c|c|c|c|c|c|c|c|c|c|}
\hline ON & 56 & 100 & 50 & 138 & 86 & -26 & -47 & -3 & 87 & 148 & 14 & 84 & 121 & 65 & 65 \\
\hline ON & 57 & 156 & 252 & 265 & 125 & -13 & 39 & -4 & 93 & 346 & 100 & 26 & 79 & 124 & 114 \\
\hline ON & 58 & 180 & 287 & 335 & 248 & 90 & 110 & -4 & 196 & 241 & 139 & 263 & 283 & 201 & 98 \\
\hline ON & 59 & 170 & 204 & 286 & 182 & 101 & 98 & -8 & 223 & 150 & 193 & 277 & 228 & 178 & 82 \\
\hline ON & 60 & 41 & 71 & 237 & 186 & 122 & 90 & -3 & 100 & 120 & 338 & 82 & 55 & 118 & 93 \\
\hline ON & 61 & 14 & 38 & 184 & 119 & 116 & 24 & 4 & 106 & 145 & 307 & 102 & 45 & 98 & 86 \\
\hline ON & 62 & 7 & 54 & 165 & 114 & 140 & 35 & 6 & 66 & 122 & 85 & 32 & 68 & 73 & 52 \\
\hline
\end{tabular}

